\title{
Aspectos microestruturais e do comportamento mecânico de revestimento duro depositado pelo processo arame tubular
}

\author{
Microstructural aspects and mechanical behavior \\ of hardfacing deposited by FCAW
}

\author{
Arley Henrique de Souza Luz ${ }^{1}$, Felipe Ribeiro Teixeira ${ }^{1}$, \\ Marco Antônio Beltrão Pamplona Junior ${ }^{1}$, Carlos Alberto Mendes da Mota ${ }^{1}$, \\ Alexandre Saldanha do Nascimento ${ }^{1}$
}

\footnotetext{
${ }^{1}$ Universidade Federal do Pará, PPGEM/GETSOLDA/UFPA, CEP: 66075-110, Rua Augusto Corrêa, Belém, PA, Brasil. e-mail: arleyhenri@gmail.com, teixeira.304@hotmail.com,pamplonam01@gmail.com, cmota@ufpa.br, saldanha77@yahoo.com.br
}

\begin{abstract}
RESUMO
Diversos tipos de revestimentos duros com materiais especiais depositados por soldagem têm sido utilizados como um método de combate ao desgaste abrasivo para aumentar a vida útil de equipamentos e reduzir custos com paradas para manutenção não programada. Este trabalho avaliou a incidência de trincas, as características microestruturais e o comportamento mecânico de revestimento duro das ligas $\mathrm{FeCrC}$ e $\mathrm{FeCrC}+\mathrm{Nb}$, depositados sobre chapas de aço AISI 1020 pelo processo de soldagem arame tubular auto protegido. Para cada liga estudada foram depositados quatro revestimentos, sendo dois de uma camada e dois de duas camadas, em dois níveis de temperatura de pré-aquecimento, à temperatura ambiente e à $250{ }^{\circ} \mathrm{C}$. Pelo ensaio de líquido penetrante, observou-se uma menor incidência de trincas para a liga $\mathrm{FeCrC}+\mathrm{Nb}$. Os resultados indicaram ainda uma microestrutura do revestimento da liga $\mathrm{FeCrC}$ constituída de grandes carbonetos primários do tipo $\mathrm{M}_{7} \mathrm{C}_{3}$ com morfologia hexagonal e em forma de agulhas, imersos em uma matriz eutética de austenita mais carbonetos. Já nos revestimentos da liga $\mathrm{FeCrC}+\mathrm{Nb}$, a microestrutura se apresentou alterada conforme o número de camadas depositadas. Para o revestimento de uma camada, a microestrutura foi caracterizada pela maior presença de carbonetos primários de nióbio $(\mathrm{NbC})$ e um reduzido número de carbonetos primários do tipo $\mathrm{M}_{7} \mathrm{C}_{3}$, sendo ambos circundados pelo eutético de austenita mais carbonetos. Quanto aos revestimentos de duas camadas com a liga $\mathrm{FeCrC}+\mathrm{Nb}$, a microestrutura foi constituída por carbonetos $\mathrm{NbC}$ e por grandes e frequentes carbonetos primários $\mathrm{M}_{7} \mathrm{C}_{3}$, em sua maioria no formato de agulha e alguns com morfologia hexagonal, envolvidos por uma mistura eutética de finos carbonetos $\mathrm{M}_{7} \mathrm{C}_{3}$ e austenita. $\mathrm{O}$ depósito da liga $\mathrm{FeCrC}$ apresentou média de microdureza superior ao da liga $\mathrm{FeCrC}+\mathrm{Nb}$ por conta da predominância e morfologia de carbonetos primários $\mathrm{M}_{7} \mathrm{C}_{3}$ evidenciados e do refinamento promovido pelo $\mathrm{Nb}$.
\end{abstract}

Palavras-chave: Revestimento duro, trincas de solidificação, carbonetos.

\begin{abstract}
Several sorts of hardfacings has been used as a method of combating wear to increase equipment life cycle, reduce costs with maintenance stops, parts recovery and replacement. This work evaluates the incidence of cracking, microstructural characteristics and the mechanical behavior of $\mathrm{FeCrC}$ and $\mathrm{FeCrC}+\mathrm{Nb}$ hardfacing alloys, deposited by self-shielded flux-cored arc welding (FCAW-S) process on SAE 1020 steel sheets. Single layer and double layer coatings were produced for both alloys in two interpass temperatures conditions, at room temperature and preheat of $250^{\circ}$. By liquid penetrant inspection, a lower incidence of cracks was observed to $\mathrm{FeCrC}+$ $\mathrm{Nb}$ alloy. The microstructures of all $\mathrm{FeCrC}$ alloy coatings presented large primary $\mathrm{M}_{7} \mathrm{C}_{3}$ carbides in a hexagonal and needle-shaped morphology on a matrix of a eutectic mixture of austenite and carbides. For FeCrC $+\mathrm{Nb}$ alloy single layer coatings, a microstructure with large niobium carbides $(\mathrm{NbC})$ and few primary $\mathrm{M}_{7} \mathrm{C}_{3}$ carbides, both being surrounded by eutectic of austenite and carbides. However, for $\mathrm{FeCrC}+\mathrm{Nb}$ alloy double layer coatings, microstructures indicate $\mathrm{NbC}$ carbides along with large and frequent $\mathrm{M}_{7} \mathrm{C}_{3}$ carbides, most of them in a needleshape morphology and a few in a hexagonal shape, being surrounded by a eutectic mixture of austenite and thin $\mathrm{M}_{7} \mathrm{C}_{3}$ carbides. Microhardness was superior for $\mathrm{FeCrC}$ alloy, due to the predominance and morphology of the primary $\mathrm{M} 7 \mathrm{C} 3$ carbides shown and the refinement promoted by $\mathrm{Nb}$.
\end{abstract}


Keywords: Hardfacing, cracking, carbides.

\section{INTRODUÇÃO}

$\mathrm{O}$ fenômeno do desgaste está presente em praticamente todos os processos industriais e, em especial, naqueles que envolvem a exploração e beneficiamento de minério e seus agregados. O desgaste é definido como um dano gerado nas superfícies sólidas envolvendo perda progressiva de material, devido ao movimento relativo entre uma superfície e um material cortante [1].

Nas áreas de manutenção das indústrias, um dos maiores desafios é o combate contínuo ao desgaste de superfícies cujas metas principais são aumentar a periodicidade entre paradas de manutenção e reduzir a troca frequente de componentes. A possibilidade de reconstruir tais componentes ou de prepará-los para melhor suportar condições operacionais que exijam grandes solicitações, ou fenômenos complexos de desgaste, é constantemente estudada visando a concepção de novas técnicas que contribuam com aumento da vida útil do equipamento utilizado, e com a redução de custos durante o processamento.

A técnica de revestimento consiste na deposição de uma liga especial resistente ao desgaste numa superfície sujeita à deterioração, destacando-se pela eficiência na prevenção ao desgaste abrasivo e na recuperação de componentes. Para obtenção de revestimentos metálicos eficazes, destaca-se o revestimento duro depositado pela soldagem em múltiplos passes sobre a superfície do substrato [2].

Os processos de soldagem mais utilizados para operação de revestimentos duros são o Arame Tubular (FCAW) e o Arco Submerso, sendo o tamanho, a forma e a composição química do metal base fatores importantes na seleção do processo [2]. De uma maneira geral, as ligas para revestimento duro utilizadas neste tipo de aplicação pertencem ao sistema Fe-Cr-C destacando-se, em particular, os aços de baixa liga, aços de alto cromo austeníticos e os ferros fundidos branco de alto cromo. Os ferros fundidos branco alto cromo estão entre os materiais com maior resistência ao desgaste abrasivo, e têm sido muito empregados desde o início do século XX. O bom desempenho destas ligas contra a abrasão deriva de uma microestrutura polifásica formada principalmente por carbonetos de cromo, bastantes duros e resistentes à ação abrasiva, suportados por uma matriz austenítica ou martensítica que, por sua vez, confere uma relativa tenacidade ao material [3].

Dentre as descontinuidades mais comuns destacam-se às trincas de contração ou de solidificação, visíveis a olho nu e causadas pela baixa ductilidade ou ainda pelo baixo limite de escoamento do depósito soldado. Essas trincas são geralmente transversais, aliviam parcialmente as tensões residuais do cordão de solda, e podem se propagar sob as condições de uso do equipamento [4]. Por outro lado, sabe-se da literatura que a incidência de trincas em revestimentos não significa necessariamente uma redução na vida útil do componente em relação ao desgaste [5].

Este trabalho tem por objetivo estudar aspectos da qualidade superficial (ensaio por líquido penetrante), microestruturais (ensaios de microscopias óptica e eletrônica de varredura) e do comportamento mecânico (ensaios de dureza e microdureza) de revestimentos duros de ferro fundido branco alto cromo das ligas $\mathrm{FeCrC} \mathrm{e}$ $\mathrm{FeCrC}+\mathrm{Nb}$, depositados sobre chapas de aço carbono pelo processo de soldagem arame tubular auto protegido.

\section{MATERIAIS E MÉTODOS}

As soldas de revestimento foram depositadas sobre chapas do aço carbono AISI 1020 medindo $200 \mathrm{~mm}$ x $50 \mathrm{~mm}$ x 9,6 mm. Como metal de adição foram utilizados dois arames tubulares de ferro fundido branco alto cromo, $\mathrm{FeCrC} \mathrm{e} \mathrm{FeCrC}+\mathrm{Nb}$, os quais têm suas respectivas composições químicas mostradas na Tabela 1 .

Tabela 1: Composição química (\% em peso) dos metais de adição fornecida pelo fabricante.

\begin{tabular}{l|l|l|l|l|l}
\hline \multirow{2}{*}{ CONSUMÍVEL } & \multicolumn{5}{|c}{ COMPOSIÇÃO QUÍMICA (\% EM PESO) } \\
\cline { 2 - 7 } & $\mathbf{C}$ & $\mathbf{S i}$ & $\mathbf{C r}$ & $\mathbf{M n}$ & Nb \\
\hline $\mathrm{FeCrC}$ & 5,0 & 1,5 & 27,0 & 1,5 & - \\
\hline $\mathrm{FeCrC}+\mathrm{Nb}$ & 5,0 & 1,2 & 22,0 & 0,5 & 7,0 \\
\hline
\end{tabular}

Os parâmetros operacionais de soldagem empregados neste trabalho foram obtidos de uma condição para baixa diluição, ideal para depósitos de revestimento, selecionada por CAVALCANTE [6]. A Tabela 2 indica os parâmetros de soldagem usados nos experimentos. 
Tabela 2: Parâmetros operacionais de soldagem utilizados neste trabalho.

\begin{tabular}{c|c|c|c|c|c|c}
\hline $\begin{array}{c}\text { TENSÃO } \\
\text { (V) }\end{array}$ & $\begin{array}{c}\text { VEL. DE ALIMENTAÇÃO } \\
\text { DO ARAME (m/min) }\end{array}$ & $\begin{array}{c}\text { VEL. DE SOLDAGEM } \\
\text { (cm/min) }\end{array}$ & $\begin{array}{c}\text { CORRENTE } \\
\text { MÉDIA (A) }\end{array}$ & $\begin{array}{c}\text { DBCP } \\
\text { (mm) }\end{array}$ & $\begin{array}{c}\text { ENERGIA DE SOL- } \\
\text { DAGEM (J/mm) }\end{array}$ & $\begin{array}{c}\text { DILUIÇÃO } \\
\text { (\%) }\end{array}$ \\
\hline 28,0 & 10,0 & 30,0 & 256,0 & 40,0 & 1433,6 & 19,6 \\
\hline
\end{tabular}

Onde: $\mathrm{DBCP}=$ Distância bico de contato peça.

A soldagem de revestimento foi realizada na posição plana com uma sobreposição lateral entre os cordões de $50 \%$ e com ângulos de ataque e de trabalho iguais a $90^{\circ}$ e $0^{\circ}$, respectivamente. A Figura 1 ilustra o esquema de posicionamento da tocha para a soldagem com arame tubular auto protegido.

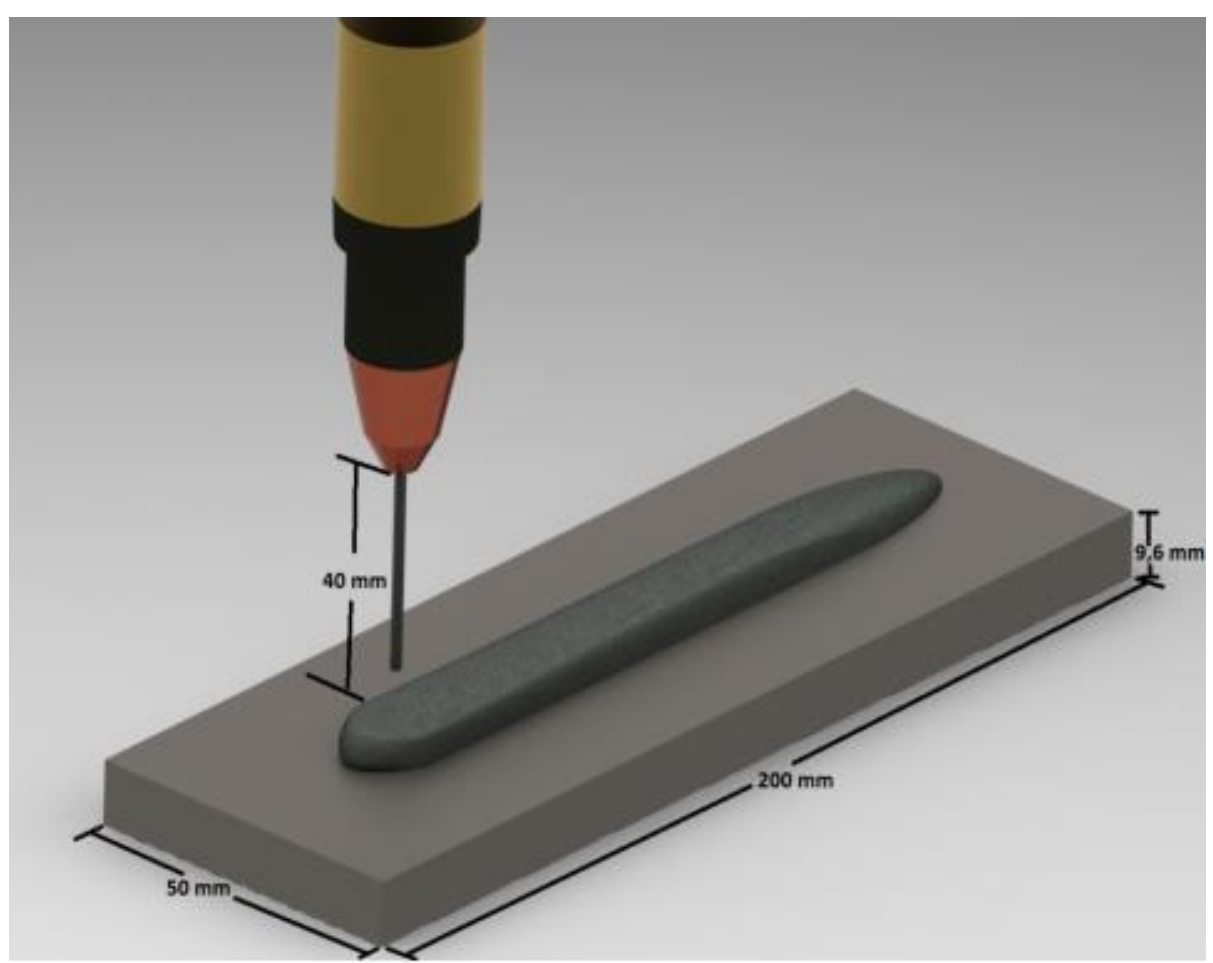

Figura 1: Esquema de posicionamento da tocha de soldagem com arame tubular auto protegido.

Foram depositados um total de oito revestimentos duros, sendo confeccionados quatro para cada arameeletrodo utilizado. Destes quatro, dois são de camada única e outros dois de dupla camada. Além disso, para cada número de camadas, foram empregadas duas condições de pré-aquecimento (temperatura ambiente e com préaquecimento de $250^{\circ} \mathrm{C}$ ).

A nomenclatura adotada para os revestimentos consistiu na seguinte lógica, partindo da esquerda para a direita: $\mathrm{FeCrC}$ ou $\mathrm{FeCrC}+\mathrm{Nb}$ para os dois arames empregados; $\mathrm{SP}$ ou $\mathrm{CP}$ indicando se o revestimento foi depositado sem ou com pré-aquecimento, respectivamente; e 1 ou 2 identificando se o revestimento apresenta uma ou duas camadas depositadas.

Após a deposição dos passes de solda, os revestimentos gerados foram submetidos ao ensaio por líquido penetrante para a análise quantitativa do número de trincas superficiais. A seguir, os depósitos foram adequadamente seccionados para a retirada e preparação de amostras da seção transversal com vistas aos exames de macro e de micrografia. As amostras da seção transversal foram, em seguida, lixadas de 80 a 1200 mesh e polidas com alumina em suspensão de $0,5 \mu \mathrm{m}$. Para as análises macro e microestrutural foi realizado um ataque com Nital $7 \%$ durante $5 \mathrm{~s}$. Após a sua preparação, as superfícies foram examinadas por microscopias ótica e eletrônica de varredura.

Para análise do comportamento mecânico foram realizados ensaios de microdureza Vickers na seção transversal do revestimento por meio de três linhas de indentação L1, L2 e L3 que são 10 mm afastadas entre si, com L2 posicionada no centro dos revestimentos (Figura 2). Para a obtenção do perfil de microdureza, empregou-se uma carga de 100 gf durante $15 \mathrm{~s}$, e um espaçamento entre indentações de $0,1 \mathrm{~mm}$. 
Visando a obtenção de resultados capazes de quantificar um nível de resistência combinada entre a matriz austenítica e os carbonetos incidentes, foram também realizados seis ensaios de dureza Rockwell C. Para cada uma das linhas de microdureza estabelecidas, foram realizados dois ensaios de dureza na região central dos revestimentos. No ensaio de dureza foi aplicada uma carga de $1470 \mathrm{~N}$ num tempo de $10 \mathrm{~s}$.

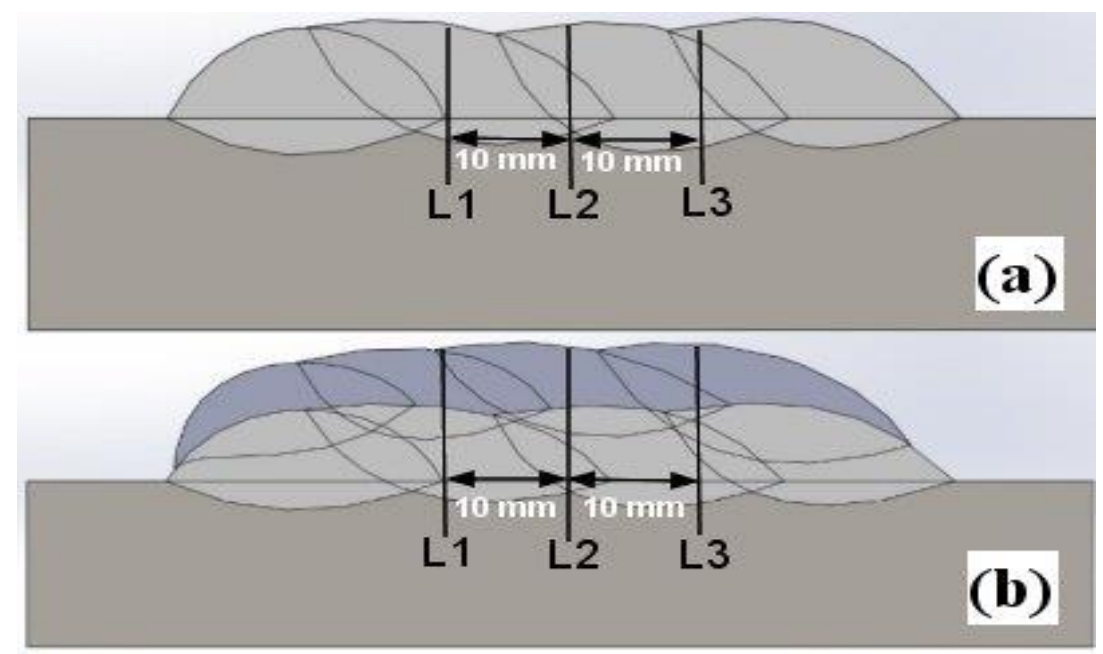

Figura 2: Linhas de indentação na seção transversal dos depósitos: (a) com uma camada; (b) com duas camadas.

\section{RESULTADOS E DISCUSSÃO}

\subsection{Aspectos superficiais e macrográficos}

A Figura 3 apresenta o resultado do ensaio de líquido penetrante, mostrando a presença de trincas transversais visíveis que afloram à superfície dos revestimentos duros depositados pelas duas ligas nas condições estabelecidas neste trabalho. A Tabela 3, por sua vez, indica o número de trincas de contração incidentes nos revestimentos de uma e duas camadas.

Os dados da Tabela 3 mostram um efeito significativo da temperatura na formação de trincas nos depósitos de revestimento duro. Nota-se, por esta tabela, uma menor incidência de trincas nos revestimentos depositados com pré-aquecimento à temperatura de $250{ }^{\circ} \mathrm{C}$, independente do arame-eletrodo ou do número de camadas depositadas. Neste caso, pode-se inferir que a imposição do pré-aquecimento promoveu reduções na taxa de resfriamento e no nível das tensões residuais e, consequentemente, contribuiu para a redução do número de trincas [7]. Para os resultados da Tabela 3 da liga $\mathrm{FeCrC}+\mathrm{Nb}$, verifica-se que os depósitos em duas camadas apresentaram uma maior incidência de trincas em relação aos depósitos com uma camada. Conforme reportado pela literatura [8], quanto maior o número de passes depositados maior será a quantidade de ciclos térmicos adicionados, os quais são responsáveis pelo aumento de tensões residuais e, por consequência, implicam numa maior susceptibilidade às trincas.

Ainda pelo exame da Tabela 3 e confrontando os resultados para as duas ligas estudadas, observa-se uma maior quantidade de trincas para a liga $\mathrm{FeCrC}$. Sabe-se que estruturas mais refinadas são menos propícias à propagação de trincas de solidificação já que podem deformar e acomodar tensões de contração mais facilmente [89]. O Nb é reconhecido como um elemento nucleador e refinador de carbonetos, assim, conforme será abordado na seção 3.2 deste trabalho, os depósitos da liga $\mathrm{FeCrC}+\mathrm{Nb}$ resultaram em uma microestrutura mais refinada composta por carbonetos primários $\mathrm{M}_{7} \mathrm{C}_{3}$ e, portanto, menos sujeita a ocorrência de trincas de solidificação. 


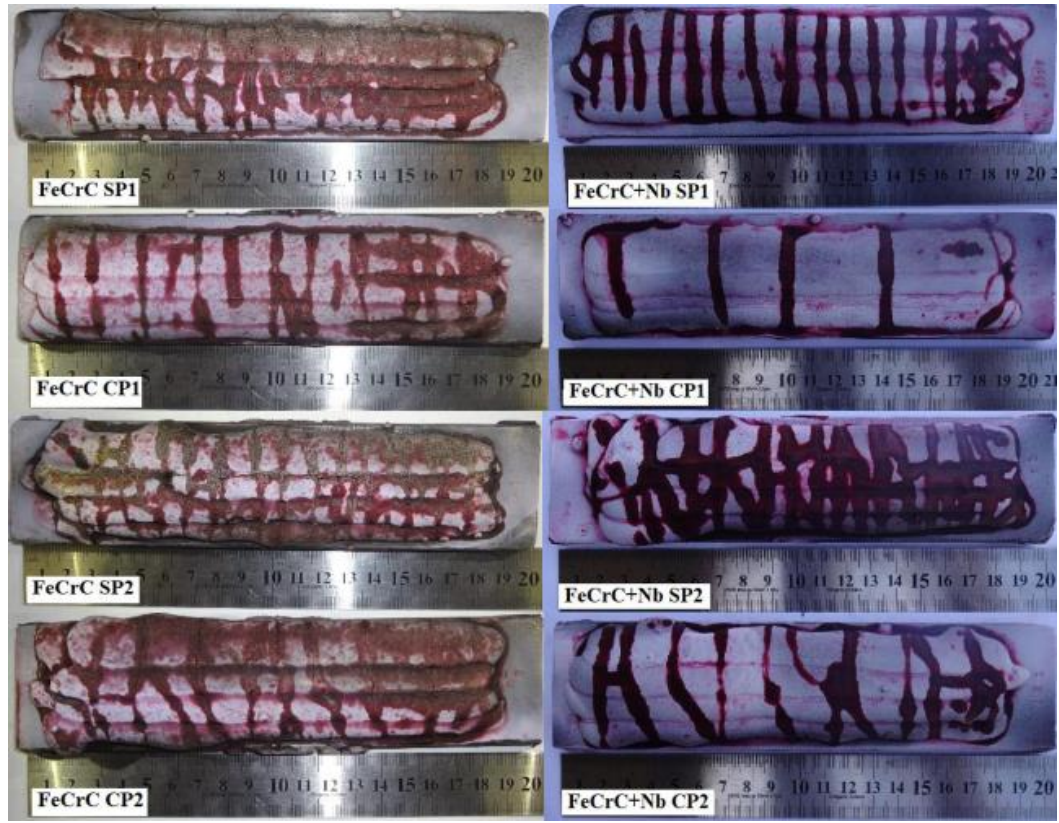

Figura 3: Resultado do ensaio de líquido penetrante. Revestimentos das ligas $\mathrm{FeCrC}$ e $\mathrm{FeCrC}+\mathrm{Nb}$, em uma e duas camadas.

Tabela 3: Número de trincas incidente do revestimento duro.

\begin{tabular}{c|c|c|c}
\hline REVESTIMENTO & №. DE TRINCAS TRANSVERSAIS & REVESTIMENTO & №. DE TRINCAS TRANSVERSAIS \\
\hline FeCrC SP1 & 20 & FeCrC+Nb SP1 & 15 \\
\hline FeCrC CP1 & 13 & FeCrC+Nb CP1 & 4 \\
\hline FeCrC SP2 & 18 & FeCrC+Nb SP2 & 17 \\
\hline FeCrC CP2 & 12 & FeCrC+Nb CP2 & 10 \\
\hline
\end{tabular}

A Figura 4 ilustra a macrografia da seção transversal dos revestimentos duros, em uma e duas camadas. Por esta figura observa-se a presença de porosidade e de trincas no metal depositado. Novamente verifica-se a redução no número de trincas para os revestimentos depositados com pré-aquecimento a $250{ }^{\circ} \mathrm{C}$, devido à redução das tensões residuais. Além disso, também é observada uma maior tendência de susceptibilidade às trincas para o arame $\mathrm{FeCrC}$, conforme já mencionado.

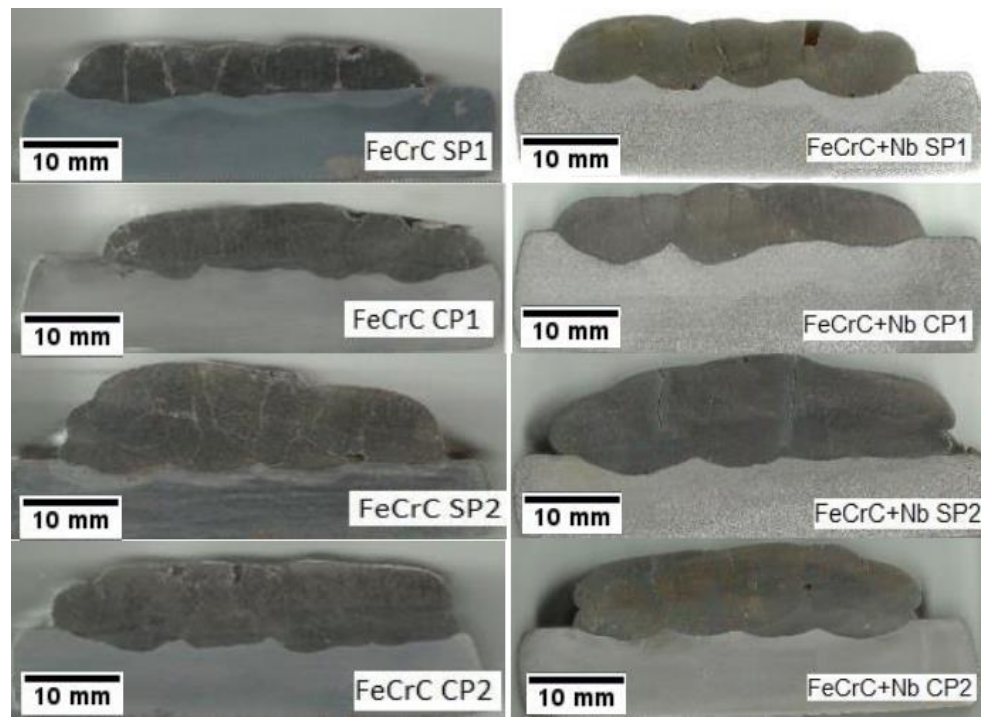

Figura 4: Macrografia da seção transversal dos revestimentos duros, em uma e duas camadas. 


\subsection{Microestrutura do revestimento duro}

Na Figura 5 são apresentadas as microestruturas da interface metal de solda (MS)/zona termicamente afetada (ZTA) para os revestimentos estudados neste trabalho.

Para a liga $\mathrm{FeCrC}$ é observada a formação de dendritas de austenita sobre a linha de fusão, e de matriz eutética de austenita mais carbonetos. Para a liga $\mathrm{FeCrC}+\mathrm{Nb}$ a microestrutura mostra a formação de dendritas de austenita adjacente à linha de fusão, e de carbonetos de $\mathrm{Nb}$ em matriz eutética. As microestruturas evidenciadas condizem com as reportadas pela literatura, a qual concluiu que as ligas do sistema $\mathrm{FeCrC}+\mathrm{Nb}$ apresentam basicamente o mesmo tipo de contraste microestrutural das ligas $\mathrm{FeCrC}$, formando-se dendritas de austenita ou carbonetos massivos $\mathrm{M}_{7} \mathrm{C}_{3}$, além da presença adicional de carbonetos $\mathrm{NbC}$ na forma primária e/ou em frações mais finas, provenientes de reações mais complexas do sistema quaternário [10-11].

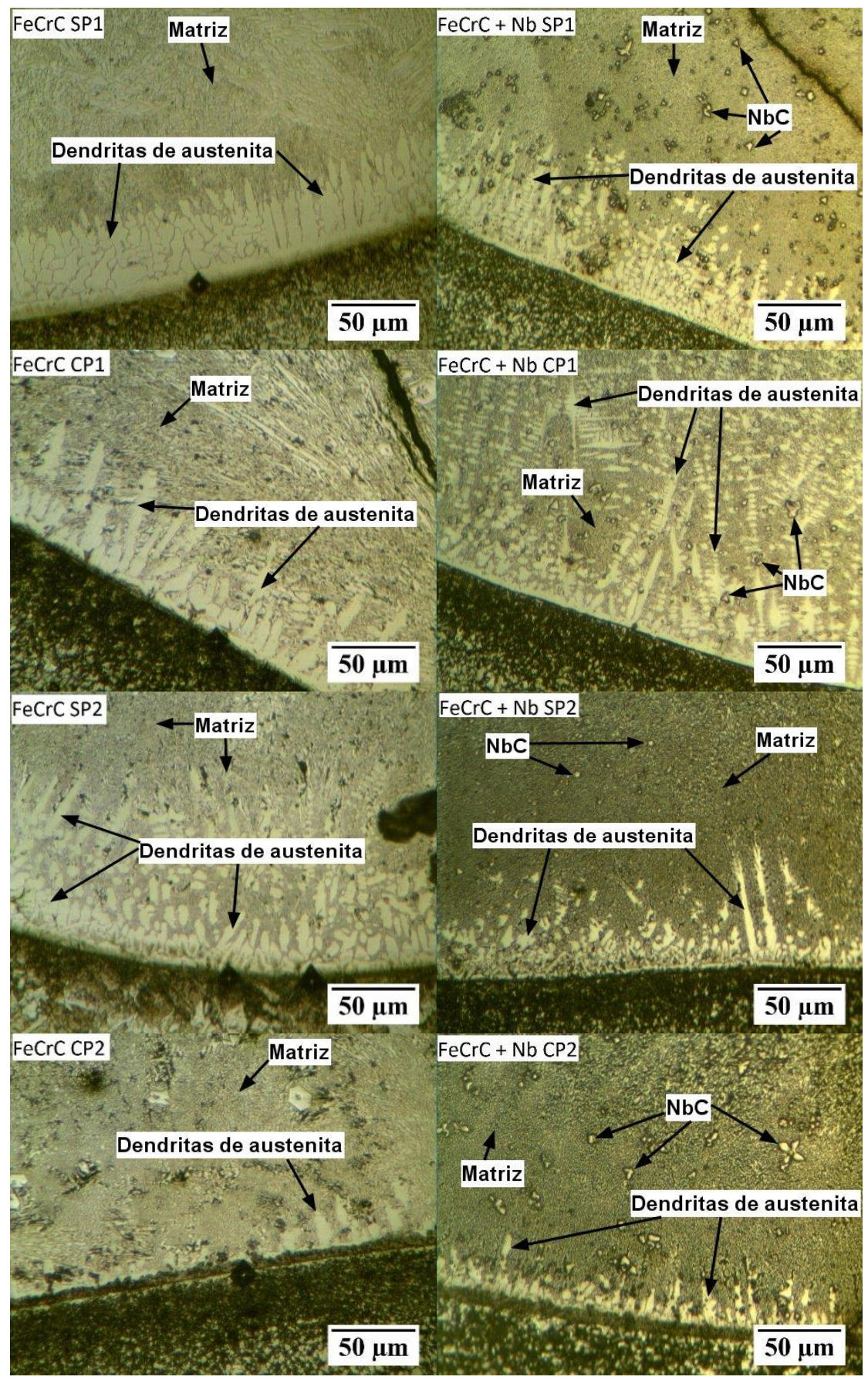

Figura 5: Microestrutura da interface MS/ZTA. Revestimentos duros das ligas $\mathrm{FeCrC} \mathrm{e} \mathrm{FeCrC}+\mathrm{Nb}$. 
A Figura 6 apresenta a microestrutura formada no centro do revestimento duro de uma e duas camadas.

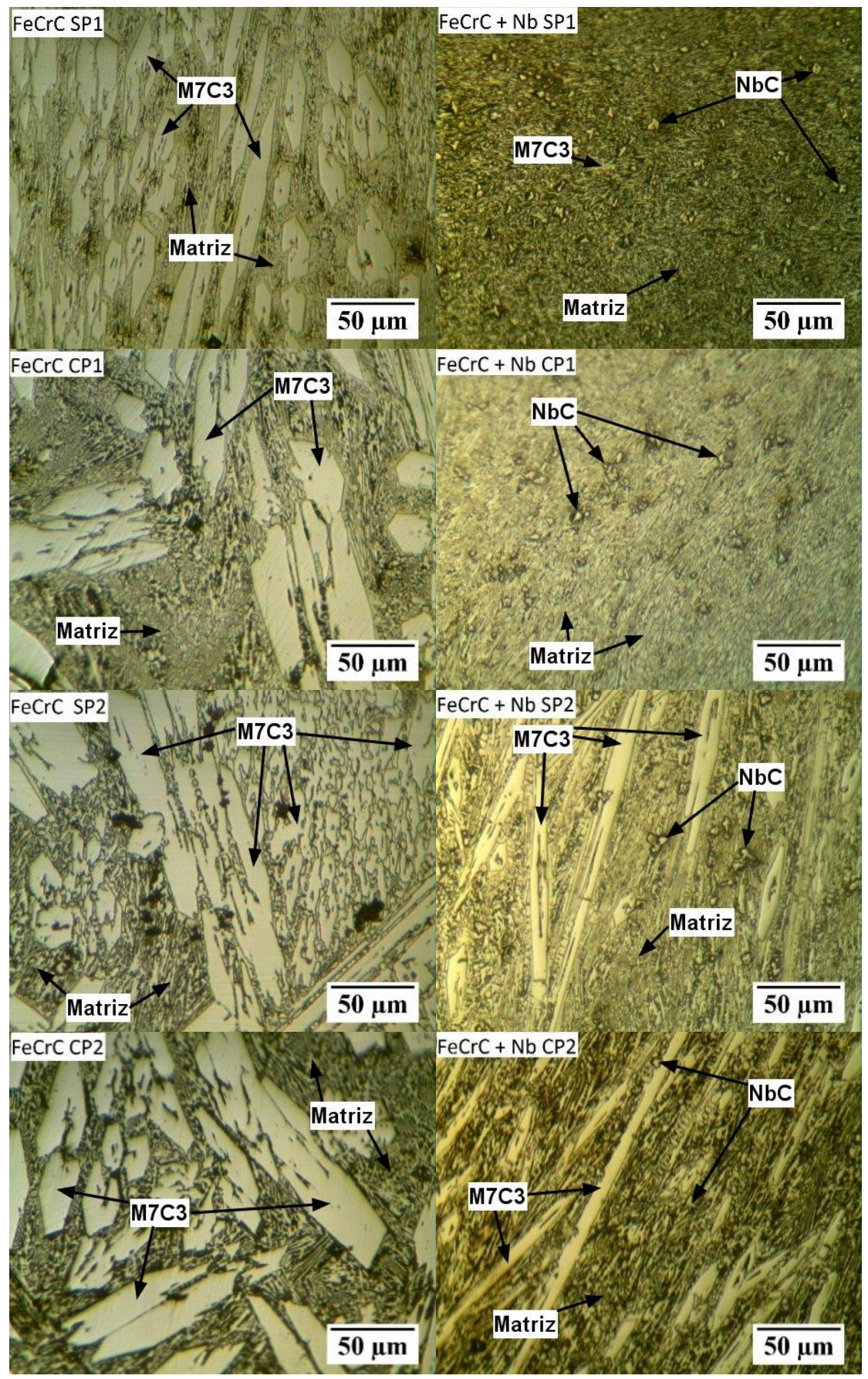

Figura 6: Microestrutura do centro do revestimento para as ligas $\mathrm{FeCrC}$ e $\mathrm{FeCrC}+\mathrm{Nb}$.

De um modo geral, e de forma similar ao verificado por outros autores [10-14], a microestrutura no centro do cordão observada na Figura 6 de todos os revestimentos da liga $\mathrm{FeCrC}$ apresentaram a formação de grandes carbonetos primários do tipo $\mathrm{M}_{7} \mathrm{C}_{3}$ com morfologia hexagonal e em forma de agulhas, imersos em uma matriz eutética de austenita mais carbonetos.

Apesar disso, para a liga $\mathrm{FeCrC}+\mathrm{Nb}$, são observadas microestruturas bastante distintas na região central do cordão entre os revestimentos de uma e duas camadas da Figura 6. Para os revestimentos de uma camada nota-se uma microestrutura caracterizada pela presença massiva de carbonetos primários de nióbio ( $\mathrm{NbC}$ ) contra pouquíssimos carbonetos primários do tipo $\mathrm{M}_{7} \mathrm{C}_{3}$, sendo ambos circundados pelo eutético de austenita mais carbonetos. Entretanto, para os revestimentos de duas camadas com a liga $\mathrm{FeCrC}+\mathrm{Nb}$, as microestruturas na parte central dos cordão indicam carbonetos $\mathrm{NbC}$ juntamente com grandes e frequentes carbonetos primários 
$\mathrm{M}_{7} \mathrm{C}_{3}$, em sua maioria no formato de agulha e alguns com morfologia hexagonal, envolvidos por uma mistura eutética de finos carbonetos $\mathrm{M}_{7} \mathrm{C}_{3}$ e austenita.

Segundo BUSHELY et al. e ATAMERT \& BHADESHIA [14-15], fases microestruturais hipoeutéticas podem ser obtidas da soldagem de ligas hipereutéticas como consequência da diluição estabelecida durante o processo de soldagem. Apesar da microestrutura dos revestimentos $\mathrm{FeCrC}+\mathrm{Nb} \mathrm{SP} 1$ e $\mathrm{FeCrC}+\mathrm{Nb} \mathrm{CP} 1$ não ser caracterizada como hipoeutética, admite-se que a mistura parcial entre o metal de solda e o substrato, e ainda, o alto nível de difusividade do carbono, podem levar a uma migração acentuada de carbono do metal de solda em direção ao metal de base, inibindo consideravelmente a formação dos carbonetos $\mathrm{M}_{7} \mathrm{C}_{3}$, haja vista que preferencialmente os carbonetos $\mathrm{NbC}$ irão se precipitar. Neste sentido, quando comparados os revestimentos de $\mathrm{FeCrC}+\mathrm{Nb}$ de uma e duas camadas, verifica-se uma pequena incidência de carbonetos primários $\mathrm{M}_{7} \mathrm{C}_{3}$ para os revestimentos de uma camada, que é atribuída a uma provável migragação de carbono do metal de solda em direção ao substrato.

Ainda pela análise da Figura 6 e comparando as micrografias das duas ligas aqui estudadas, verifica-se claramente a presença de carbonetos primários $\mathrm{M}_{7} \mathrm{C}_{3}$ em um maior tamanho e frequência para a liga $\mathrm{FeCrC}$. De acordo com ZHI et al. [16] e CHATTERJEE \& PAL [17], este resultado se deve à adição de Nb na liga a $\mathrm{FeCrC}+\mathrm{Nb}$, haja vista que este elemento reduz a quantidade de carbono no metal líquido devido a formação dos primeiros precipitados de $\mathrm{NbC}$, o que diminui, assim, a fração volumétrica e o tamanho dos carbonetos primários $\mathrm{M}_{7} \mathrm{C}_{3}$.

Visando um melhor contraste entre as fases presentes para ambas as ligas, a Figura 7 apresenta micrografias via MEV (microscópio eletrônico de varredura) dos revestimentos duros de duas camadas com préaquecimento a $250{ }^{\circ} \mathrm{C}$. Na Figura 7(a) destaca-se a presença dos carbonetos $\mathrm{M}_{7} \mathrm{C}_{3}$ grosseiros, partículas cinza claro, dispersos na matriz da liga de $\mathrm{FeCrC}$. Durante o início da solidificação desta liga, regiões ricas em $\mathrm{Cr}$ e $\mathrm{C}$ se estabelecem gerando núcleos para a precipitação de carbonetos. Estas partículas dispõem de facilidade para crescer aleatoriamente no seio do metal de solda ainda líquido, consumindo significativas quantidades de cromo e de carbono. Com a diminuição de soluto devido a formação de carbonetos primários, a austenita remanescente nos bolsões interdendríticos mantem-se relativamente pobre em carbono, mas ainda em quantidade suficiente para a precipitação de carbonetos do tipo $\mathrm{M}_{7} \mathrm{C}_{3}$ durante a transformação eutética. Logo, a microestrutura final dos revestimentos $\mathrm{FeCrC}$ seria composta por carbonetos primários grosseiros com morfologia prismática, muitas vezes agrupados em feixes, que estariam envoltos por uma solução sólida substitucional ferro-cromo contendo carbonetos mais finos [18].
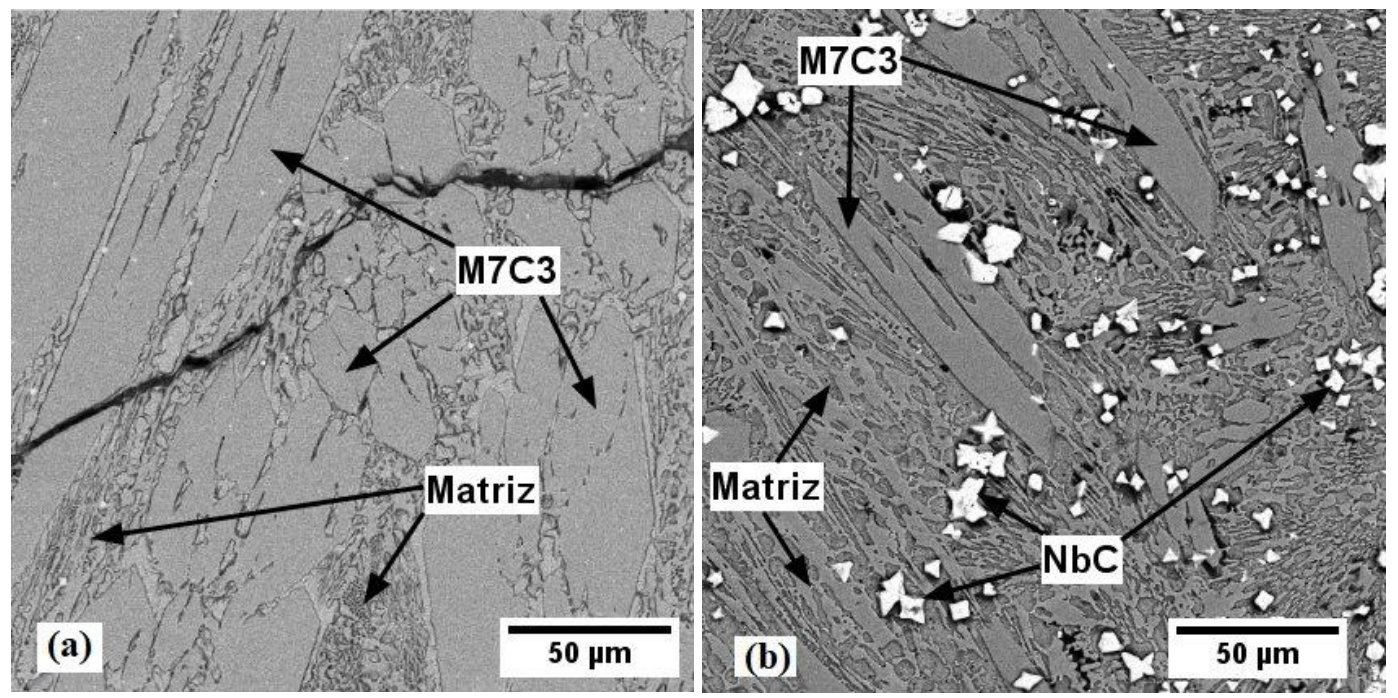

Figura 7: Micrografia via MEV de ambas as ligas (a) $\mathrm{FeCrC}$ (a) $\mathrm{FeCrC}+\mathrm{Nb}$

Para a liga $\mathrm{FeCrC}+\mathrm{Nb}$ da Figura 7(b) é verificada a presença dos carbonetos NbC, partículas brancas, e $\mathrm{M}_{7} \mathrm{C}_{3}$, partículas cinza claro, dispersos em matriz eutética. Conforme já comentado, a primeira fase sólida formada durante a solidificação desta liga consiste no carboneto $\mathrm{NbC}$. Com a formação de $\mathrm{NbC}$, zonas ricas em Fe e Cr são estabelecidas, as quais aumentam a temperatura de formação de austenita e se comportam como núcleos para formação da mesma. Desde que o coeficiente de partição de $\mathrm{Cr}$ e $\mathrm{C}$ em $\gamma$-Fe seja menor que 1 , estes dois elementos passam a segregar em áreas adjacentes durante a formação da austenita, possibilitando que a região compreendida entre o $\mathrm{NbC}$ e a austenita permaneça enriquecida de $\mathrm{Cr}$ e $\mathrm{C}$. Por outro lado, devido ao 
rápido resfriamento dos depósitos de solda, caractarístico da operação de soldagem, não há tempo suficiente para a difusão de $\mathrm{Cr}$ e $\mathrm{C}$ se estabelecendo, então, uma condição propícia à nucleação do carboneto de cromo no entorno e adjacente ao $\mathrm{NbC}$. Assim, os dois carbonetos podem ser nucleados de forma aglomerada [19]. Em relação aos carbonetos $\mathrm{M}_{7} \mathrm{C}_{3}$, apesar de majoritariamente se apresentarem na forma de agulha, pode-se notar a sua presença na forma hexagonal para ambos as ligas indicando o crescimento destes carbonetos também na direção longitudinal.

\subsection{Comportamento mecânico do revestimento duro}

A Figura 8 apresenta o resultado de microdureza para os revestimentos duros de uma e duas camadas. Destacamse as linhas horizontais vermelhas, que indicam as médias de microdureza obtidas do metal de solda de cada liga.

Em termos dos resultados de microdureza expressos pela média dos valores obtidos, tem-se que a liga $\mathrm{FeCrC}$ apresentou um valor médio de $1150 \mathrm{HV}$, enquanto que a liga $\mathrm{FeCrC}+\mathrm{Nb}$ obteve um valor médio de $900 \mathrm{HV}$. Segundo WANG e LI [18], os carbonetos primários $\mathrm{M}_{7} \mathrm{C}_{3}$ apresentam dureza de até $1800 \mathrm{HV}$ e os carbonetos $\mathrm{NbC}$ dispõem de dureza de até $2500 \mathrm{HV}$. Assim, apesar dos carbonetos $\mathrm{NbC}$ serem mais duros do que os carbonetos $\mathrm{M}_{7} \mathrm{C}_{3}$, foi verificada uma redução na média de microdureza para a liga $\mathrm{FeCrC}+\mathrm{Nb}$. Isto se justifica devido ao efeito combinado entre o pequeno tamanho e a dispersão considerável encontrada para os carbonetos $\mathrm{NbC}$ na matriz, e ainda, aos menores tamanho e quantidade dos carbonetos primários $\mathrm{M}_{7} \mathrm{C}_{3}$ encontrados para a liga $\mathrm{FeCrC}+\mathrm{Nb}$. Além disso, a metodologia utilizada para o ensaio de microdureza também é um fator de influência sobre os valores encontrados, haja vista que as indentações não foram pontuais sobre os carbonetos, mas seguiram uma sequência linear, podendo atingir aleatoriamente tanto a matriz eutética quanto os carbonetos.

Ainda pelo exame da Figura 8, observa-se um grande desvio padrão, associado a uma considerável dispersão entre os resultados de microdureza, e que é atribuído à diferença de resistência entre a matriz eutética (em cerca de $900 \mathrm{HV}$ ) e os carbonetos primários $\mathrm{M}_{7} \mathrm{C}_{3}$ e de $\mathrm{NbC}$. Além disso, observa-se uma maior dispersão no desvio padrão dos valores de microdureza para a liga $\mathrm{FeCrC}$ em relação a liga $\mathrm{FeCrC}+\mathrm{Nb}$, justificada pela menor presença e tamanho de carbonetos primários $\mathrm{M}_{7} \mathrm{C}_{3}$ para a liga com adição de $\mathrm{Nb}$.

Segundo MACEDO e GALLEGO [20], o comportamento desejável ao perfil de microdureza ao longo da seção transversal de revestimentos duros deve ser de decaimento contínuo e suave no sentido superfície-metal de base, sem oscilações bruscas. Neste sentido, por apresentar as menores dispersões nos valores de microdureza, pode-se afirmar que diante das condições estabelecidas neste trabalho a liga $\mathrm{FeCrC}+\mathrm{Nb}$ é a mais indicada para a aplicação em revestimento duro.

Conforme já comentado, seis ensaios de dureza Rockwell $\mathrm{C}$ foram realizados visando se alcançar resultados capazes de quantificar um nível de resistência combinada entre a matriz e os carbonetos, sendo realizados dois ensaios de dureza na região central dos revestimentos ao longo de cada uma das linhas de microdureza estabelecidas. A Figura 9 apresenta os resultados médios de dureza para cada condição de revestimento com seus respectivos desvios padrões.

Apesar da diferença significativa das médias de microdureza obtidas no revestimento das ligas empregadas neste trabalho, a análise da Figura 9 não indica variações discrepantes nos valores de dureza entre os revestimentos das ligas $\mathrm{FeCrC}$ e $\mathrm{FeCrC}+\mathrm{Nb}$, independentemente das condições de pré-aquecimento ou do número de camadas depositadas 

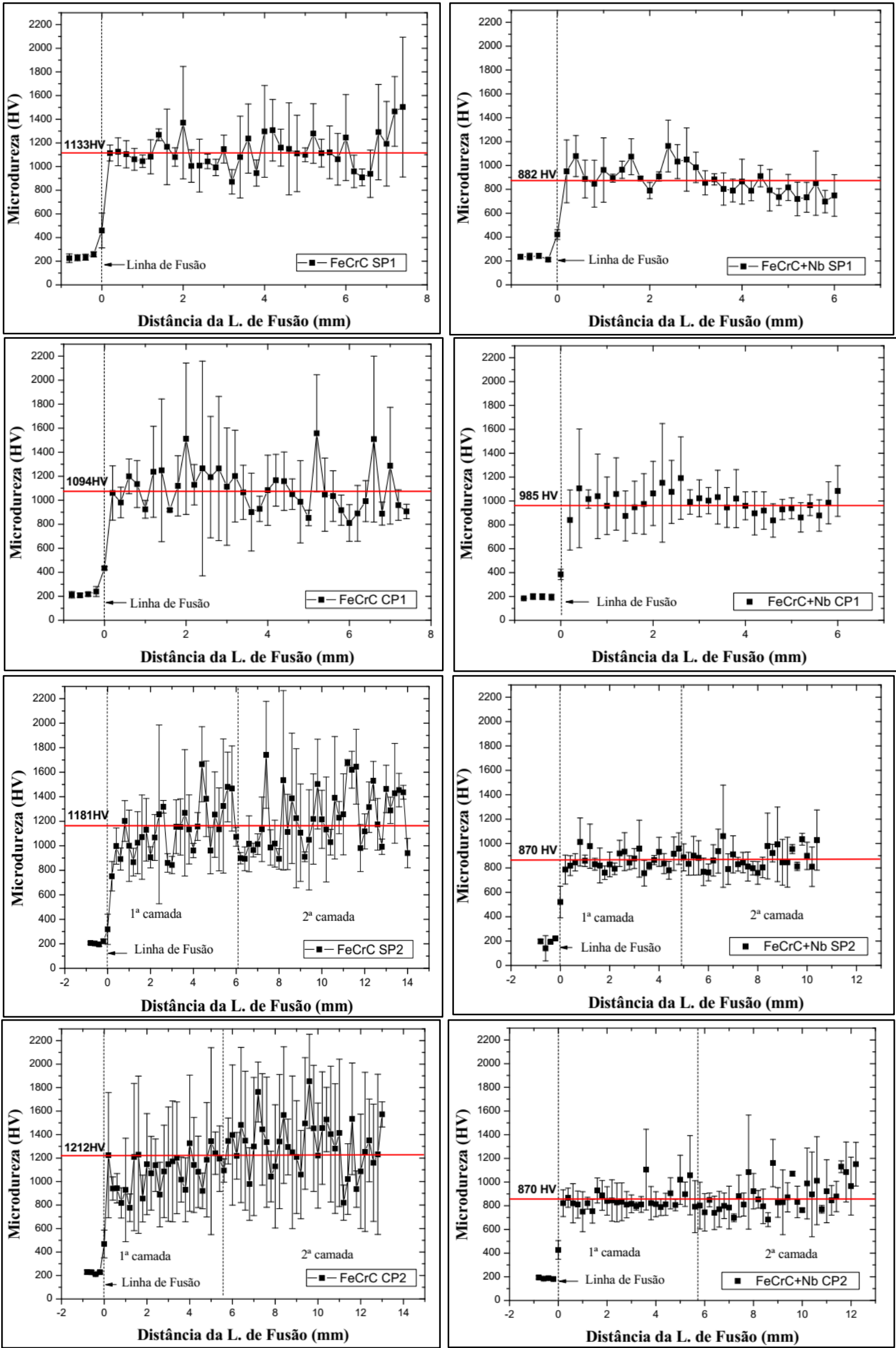

Figura 8: Resultados de microdureza para os revestimentos em uma e duas camadas. 


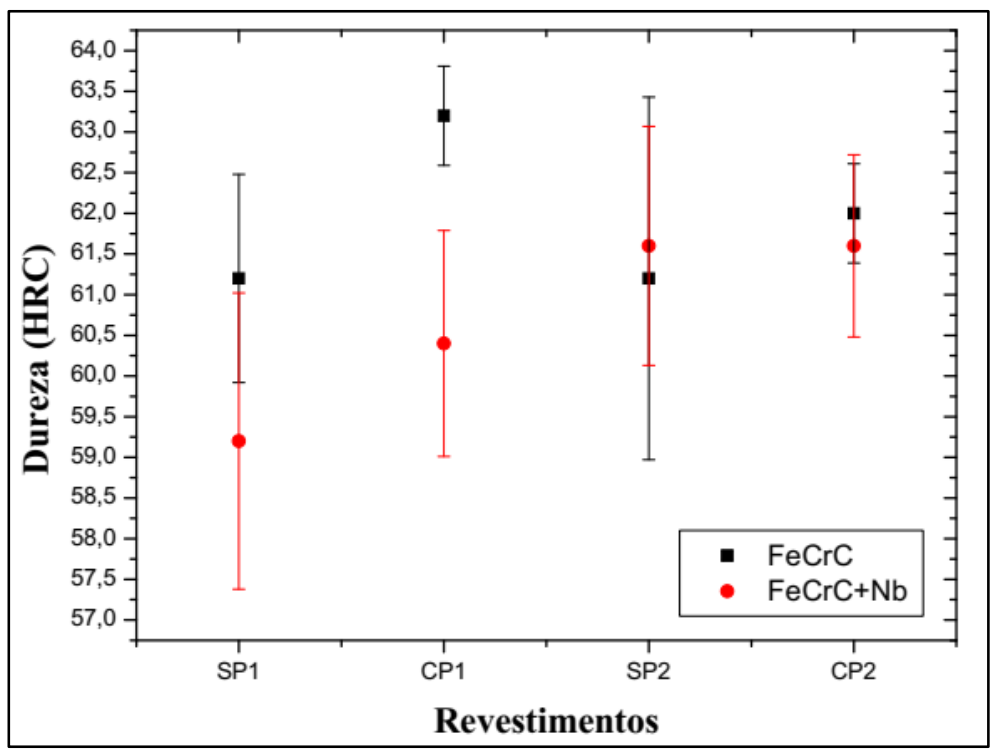

Figura 9: Resultados de dureza para os revestimentos em uma e duas camadas.

\section{CONCLUSÕES}

A temperatura de pré-aquecimento afetou o número de trincas nos revestimentos de tal forma que o uso de préaquecimento a $250{ }^{\circ} \mathrm{C}$ reduziu a incidência de trincas de solidificação no metal depositado.

A incidência de trincas no revestimento da liga $\mathrm{FeCrC}+\mathrm{Nb}$ foi menor devido ao refinamento da microestrutura pela adição de $\mathrm{Nb}$, e aumentou conforme do número de camadas depositadas.

A incidência de trincas no revestimento da liga $\mathrm{FeCrC}$ não variou em função do número camadas depositadas.

Em termos da microestrutura próxima à linha de fusão, observou-se para a liga $\mathrm{FeCrC}$ a formação de dendritas de austenita sobre a linha de fusão e uma matriz eutética de austenita mais carbonetos, enquanto que para a liga $\mathrm{FeCrC}+\mathrm{Nb}$ a microestrutura mostra a formação de dendritas de austenita adjacentes à linha de fusão e de carbonetos de $\mathrm{Nb}$ em matriz eutética.

$\mathrm{O}$ revestimento duro da liga $\mathrm{FeCrC}$ apresentou no centro do cordão a formação de grandes carbonetos primários do tipo $\mathrm{M}_{7} \mathrm{C}_{3}$ com morfologias hexagonal e em agulhas, imersos em uma matriz eutética de austenita mais carbonetos.

No revestimento de uma camada da liga $\mathrm{FeCrC}+\mathrm{Nb}$ a microestrutura se caracterizou pela presença predominante de carbonetos primários $\mathrm{NbC}$ e pouquíssimos carbonetos primários do tipo $\mathrm{M}_{7} \mathrm{C}_{3}$, sendo ambos circundados pelo eutético de austenita mais carbonetos.

Nos revestimentos de duas camadas da liga $\mathrm{FeCrC}+\mathrm{Nb}$ a microestrutura indica a presença de carbonetos de nióbio, juntamente com grandes e frequentes carbonetos primários $\mathrm{M}_{7} \mathrm{C}_{3}$, majoritários na forma de agulhas e alguns com morfologia hexagonal, envolvidos por uma mistura eutética de finos carbonetos $\mathrm{M}_{7} \mathrm{C}_{3}$ e austenita.

A formação de carbonetos primários $\mathrm{M}_{7} \mathrm{C}_{3}$ em maiores tamanho e frequência para a liga $\mathrm{FeCrC}$, e ainda, o pequeno tamanho e a dispersão considerável dos carbonetos $\mathrm{NbC}$ para a liga $\mathrm{FeCrC}+\mathrm{Nb}$, justificam a menor média de microdureza obtida para a liga com adição de $\mathrm{Nb}$.

\section{AGRADECIMENTOS}

À Vale, pelo financiamento da pesquisa. Ao GETSOLDA/UFPA, ao GPEMAT/UFPA e ao IFPA, pela disponibilidade de equipamentos.

\section{BIBLIOGRAFIA}

[1] ZUM GAHR, K. J., Microstructure and wear of materials, Tribology series v10, 1 ed., Amsterdam, Elsevier, 1987.

[2] CORRÊA, E. O., Avaliação da resistência ao desgaste de ligas desenvolvidas para solda de revestimento duro para uso sob condições altamente abrasivas, Tese de D.Sc., Universidade Federal de São Carlos, São Carlos, RJ, Brasil, 2005. 
[3] GREGOLIN, J.A.R., Desenvolvimento de ligas Fe-C-Cr-Nb resistentes ao desgaste, Tese de D.Sc., UNICAMP, Campinas, SP,Brasil, 1990.

[4] WAINER, E., BRANDI, S. D., DE MELLO, F. D. H., Soldagem - Processos e Metalurgia, 4 ed., São Paulo, Edgard Blücher Ltda, 1992.

[5] SCOTTI, A., ROSA, L. A. A., "Influence of oscillation parameters on crack formation in automatic Fe-B hardafacing”, Journal of Materials Processing Technology, v. 65, pp. 272-280, 1997.

[6] CAVALCANTE, H. dos S, Levantamento dos parâmetros operacionais de soldagem para aplicação de revestimento duro pelo processo de soldagem FCAW, Trabalho de Conclusão de Curso, UFPA, Belém, PA, Brasil, 2015 .

[7] KOU, S., Welding Metallurgy, 2 ed., New Jersey, John Wiley e Sons, 2002.

[8] DVORNAK, M. J., FROST, R. H., OLSON, D. L., "Influence of solidification kinetics on aluminum weld grain refinement”, Welding Journal, v. 70, n. 10, pp. 271-276, Oct. 1991.

[9] HERNÁNDEZ, O. J. S., Otimização do Consumível na Solda de Revestimento Aplicada na Indústria Sucroalcooleira, Dissertação de Msc., Universidade Federal de São Carlos, São Carlos, RJ, Brasil, 1997.

[10] LIMA, A. C., Estudo da aplicação de revestimento duro por soldagem com arames tubulares quanto à resistência ao desgaste de facas picadoras de cana-de-açúcar. Tese de D.Sc., Universidade Federal de Uberlândia, Uberlândia, MG, Brasil, 2008.

[11] LIMA, A. C., FERRARESI, V. A., REIS, R. P, "Performance Analysis of Weld Hardfacings Used in the Sugar/Alcohol Industry”, Journal of Materials Engineering and Performance, v. 23, pp. 1823-1833, 2014.

[12] SOUZA, D. D. B. G., FERRARESI, V. A., “Aplicação de revestimento duro utilizando o processo FCAW duplo arame para diferentes tipos de consumíveis utilizados na indústria sucroalcooleira”, Revista Matéria, v. 22 , n.1, 2017.

[13] CRESPO, A. C., FUENTES, R. F., FERRARESI, V. A., GONÇALVES, R. A., SCOTTI, A. "Microstructure and Abrasion Resistance of Fe-Cr-C and Fe-Cr-C-Nb Hardfacing Alloys Deposited by S-FCAW and Cold Solid Wires", Revista Soldagem \& Inspeção, v.21, n.3, pp. 342-353, 2016.

[14] BUCHELY, M. F., GUTIERREZ, J.C., LEÓN, L.M., TORO, A., “The effect of microstructure on abrasive wear of hardfacing alloys", Wear, v. 259, pp. 52-61, 2005.

[15] ATAMERT, S. , BHADESHIA, H. K. D. H., "Microstructure and stability of FeCrC hardfacing alloys", Materials Science and Engineering, v. 130, pp. 101-111, 1990.

[16] ZHI, H., XING, J., FU, H., et al., "Effect of niobium on the as-cast microestruture of hypereutectic high chromium cast iron", Materials Letters, n. 62, pp.857-860, 2008.

[17] CHATTERJEE, S., PAL, T.K., "Wear Behaviour of Hardfacing Deposits on Cast Iron”, Wear. v. 255, n.4, pp. 417-425, 2003.

[18] POLIDO, R. S., BARELA, R., MACEDO, B. P. N., et al., "Microestrutura de revestimentos duros resistentes ao desgaste abrasivo empregados na indústria sucroalcooleira", In: XXIV CONSOLDA - Congresso Nacional de Soldagem. São Paulo - SP, Brasil, 18-21 Maio 2008.

[19] WANG, Q., LI, X., "Effects of Nb, V, and W on microstructure and abrasion resistence of FeCrC hardfacing alloys", Welding Journal, v. 89, pp. 133-139, 2010.

[20] MACEDO, B. P. N., GALLEGO, J., "Efeito da morfologia dos carbonetos e da variação da microdureza em revestimentos duros resistentes ao desgaste abrasivo com aplicação na indústria sucroalcooleira”, In: XVI Congresso Nacional de Estudantes de Engenharia Mecânica, Florianópolis, SC, ago. 2009.

\section{ORCID}

Arley Henrique de Souza Luz Felipe Ribeiro Teixeira Marco Antônio Beltrão Pamplona Junior Carlos Alberto Mendes da Mota Alexandre Saldanha do Nascimento https://orcid.org/0000-0002-8938-7285 https://orcid.org/0000-0002-9945-404X https://orcid.org/0000-0002-4028-7937 https://orcid.org/0000-0002-4745-1429 https://orcid.org/0000-0002-0684-3151 